\title{
Lewy body compared with Alzheimer dementia is associated with decreased functional connectivity in resting state networks
}

\author{
Eva R. Lowther ${ }^{\mathrm{a}}$, John T. O’Brien ${ }^{\mathrm{b}, \mathrm{c}}$, Michael J. Firbank ${ }^{\mathrm{b}}$, Andrew M. Blamire ${ }^{\mathrm{d}, *}$ \\ a Institute for Ageing and Health and Newcastle Magnetic Resonance Centre, Campus for Ageing and Vitality, Newcastle University, \\ Newcastle upon Tyne NE4 5PL, United Kingdom \\ ${ }^{\mathrm{b}}$ Institute for Ageing and Health, Newcastle University, Newcastle upon Tyne, United Kingdom \\ ${ }^{\mathrm{c}}$ Department of Psychiatry, University of Cambridge, Cambridge, United Kingdom \\ ${ }^{\mathrm{d}}$ Institute for Cellular Medicine and Newcastle Magnetic Resonance Centre, Newcastle University, Newcastle upon Tyne, United Kingdom
}

\section{A R T I C L E I N F O}

Article history:

Received 23 July 2013

Received in revised form

23 May 2014

Accepted 19 June 2014

Keywords:

Basal ganglia

Default mode network

Functional MRI

\begin{abstract}
A B S T R A C T
Resting state functional magnetic resonance imaging (fMRI) was used to measure whole brain functional connectivity within specific networks hypothesised to be more affected in dementia with Lewy bodies (DLB) (a disease characterised by prominent attentional deficits, spontaneous motor features of parkinsonism and depression) than in Alzheimer's disease (AD) and controls. This study involved 68 subjects (15 DLB, $13 \mathrm{AD}$ and 40 controls) who were scanned using resting state BOLD (blood-oxygenlevel-dependent) fMRI on a 3 T MRI scanner. Functional connectivity was measured using a model-free independent component analysis approach that consisted of temporally concatenating the resting state fMRI data of all study subjects and investigating group differences using a back-reconstruction procedure. Resting state functional connectivity was affected in the default mode, salience, executive and basal ganglia networks in DLB subjects compared with AD and controls. Functional connectivity was lower in DLB compared with AD and controls in these networks, except for the basal ganglia network, where connectivity was greater in DLB. No resting state networks showed less connectivity in AD compared with DLB or controls. Our results suggest that functional connectivity of resting state networks can identify differences between DLB and AD subjects that may help to explain why DLB subjects have more frequent attentional deficits, parkinsonian symptoms, and depression than those with AD.
\end{abstract}

(c) 2014 Elsevier Ireland Ltd. All rights reserved.

\section{Introduction}

Dementia with Lewy bodies (DLB) is the second most common cause of neurodegenerative dementia after Alzheimer's disease (AD) (McKeith et al., 1996, 2005; Geser et al., 2005). The clinical symptoms of DLB and AD can overlap, a fact that makes differentiating the disorders difficult. Neuroimaging is used in dementia to better understand neurobiological changes underpinning key symptoms and clinically to enhance diagnostic accuracy. Compared with the literature on $\mathrm{AD}$, few neuroimaging studies have investigated DLB, and the neural changes responsible for the distressing symptoms of attentional deficits, motor features of parkinsonism, and depression that are characteristic of DLB are not well understood.

Resting state BOLD (blood-oxygen-level-dependent) functional magnetic resonance imaging (fMRI) shows temporal correlations in spontaneous low-frequency fluctuations (SLFs) (at $<0.1 \mathrm{~Hz}$ )

\footnotetext{
* Corresponding author. Tel.: +44 191248 1159; fax: +44 1912481151

E-mail address: andrew.blamire@ncl.ac.uk (A.M. Blamire).
}

between distant but anatomically connected brain regions (Biswal et al., 1995), representing functional connectivity (Fox and Raichle, 2007). Both independent component analysis (ICA) (Beckmann et al., 2005) and seed-region (Damoiseaux et al., 2006) approaches can be used to organise brain regions into at least 10 resting state networks that plausibly represent different sensory and cognitive processes.

Initially, resting state fMRI studies focussed on the default mode network (composed of posterior cingulate, precuneus, lateral parietal, lateral temporal and medial frontal regions), which is active at rest and deactivates when a task is performed (Raichle et al., 2001). This network has been shown to be affected in AD, with abnormalities increasing as the disease progresses (Zhou et al., 2010; Zhang et al., 2011; Damoiseaux et al., 2012), but more recently it has been shown that other resting state networks are also affected in $\mathrm{AD}$, for example, the sensory motor, dorsal attention and salience networks (Brier et al., 2012; Zhou et al., 2010). Few studies have investigated functional connectivity in DLB, and those studies which have considered this group have used slightly different analytical approaches and, perhaps as a consequence, came to different conclusions. 
Previously, we investigated functional connectivity in DLB and AD using a seed-region approach and showed abnormally increased connectivity compared with findings in controls in the posterior cingulate and putamen in DLB, in the hippocampus in $\mathrm{AD}$, and in the caudate and thalamus in both DLB and AD (Kenny et al., 2011, 2013). Galvin et al. (2011) used a similar approach but focussed solely on precuneus connectivity and used the whole structure as the seed region. Their study showed both increased connectivity with putamen and parietal regions and decreased connectivity with prefrontal and primary visual cortices (Galvin et al., 2011).

In the current study, instead of measuring connectivity with predefined regions of interest, we adopted a model-free independent component analysis (ICA) approach. This approach enables investigation of whole brain functional connectivity ensuring optimal use of the study data. The ICA method was used in DLB subjects by Franciotti et al. (2012), who reported no abnormalities in default mode network connectivity in DLB but did not report analysis of other identified networks (Franciotti et al., 2012). Here we investigated the wider set of resting state networks in DLB subjects. We hypothesised that functional connectivity would be significantly altered in DLB compared with control and AD subjects within the following networks:

(a) Default mode, salience and executive networks because of the attentional deficits which are greater in DLB than AD subjects (Ballard et al., 2001).

(b) Basal ganglia and limbic networks, specifically the caudate because of its role in emotional regulation and the greater severity of depression in DLB, the putamen because structural pathology and neurotransmitter abnormalities here are associated with parkinsonian symptoms in DLB (Walker et al., 2002; O'Brien et al., 2004), and the thalamus which is involved in maintaining consciousness (Perry and Perry, 2004) and fluctuating cognition is a core feature of DLB (McKeith et al., 1996).

\section{Methods}

\subsection{Participants}

This study involved 68 subjects aged over 60 years: 15 DLB, 13 AD and 40 control subjects; the same subjects have also been investigated in previous studies (Kenny et al., 2010, 2011, 2013). DLB and AD subjects were recruited from clinical Old Age Psychiatry, Geriatric Medicine and Neurology outpatient services; controls were recruited through local advertisement or were partners of the dementia subjects. The study was approved by the local ethics committee, and all subjects gave signed informed consent for participation. DLB subjects met consensus criteria for probable DLB including the presence of two or more core clinical features (fluctuating cognition, visual hallucinations and/or parkinsonism) (McKeith et al., 1996, 2005). AD subjects fulfilled National Institute of Neurological and Communicative Diseases and Stroke/Alzheimer's Disease and Related Disorders Association (NINCDS/ADRDA) criteria for probable AD (McKhann et al., 1984). Diagnoses were made by consensus between two experienced clinicians, a method previously validated against autopsy diagnosis (McKeith et al., 2000). All of the DLB subjects who underwent ${ }^{123}$ I-labelled $N$-(3-fluoropropyl)-2ß-carbomethoxy-3ß-(4-iodophenyl) nortropane $\left({ }^{123} \mathrm{I}-\mathrm{FP}-\mathrm{CIT}\right)$ single photon emission computed tomography (SPECT) imaging during their clinical diagnostic assessment $(n=9)$ showed reduced dopamine transporter uptake in the basal ganglia consistent with their diagnosis.

Detailed physical, neurological, and neuropsychiatric examinations were carried out as follows: the Mini-Mental State Examination (MMSE) (Folstein et al., 1975) to assess cognitive status, the Geriatric Depression Scale (GDS) to assess depressive symptoms (Sheikh and Yesavage, 1986), the Neuropsychiatric Inventory (NPI) to assess neuropsychiatric symptoms (Cummings et al., 1994), the Clinical Assessment of Fluctuation Scale (CAFS) to assess fluctuating cognition (Walker et al., 2000), and the motor subsection of the Unified Parkinson's Disease Rating Scale (UPDRS III) for motor features of parkinsonism (Fahn and Elton (1987)). Exclusion criteria were severe concurrent illness (apart from dementia in the DLB and AD groups), the presence of space-occupying lesions on MRI, stroke history and any contraindications to MRI. None of the control subjects had a history of psychiatric illness. A larger control group size was used to obtain a robust depiction of the brain networks in the older brain.

\subsection{Imaging}

All subjects were scanned on the same 3 T MRI system (Intera Achieva scanner, Philips Medical System, Eindhoven, The Netherlands). An eight-channel head coil was used to collect resting state fMRI scans using a gradient-echo echo-planar imaging sequence. The scan timings and parameters were as follows: 25 axial slices, 128 volumes, anterior-posterior acquisition, in-plane resolution $=2 \times 2 \mathrm{~mm}^{2}$, slice thickness $=6 \mathrm{~mm}$, repetition time $=3000 \mathrm{~ms}$, echo time $=40 \mathrm{~ms}$, field of view $=260$ $\times 150 \times 260 \mathrm{~mm}^{3}$, acquisition time $=6.65 \mathrm{~min}$. Conventional structural 3D T1weighted scans were also collected and used for co-registration of the functional scans.

\subsection{Resting state fMRI analysis}

\subsubsection{Data pre-processing}

Data were analysed using the FMRIB's Software Library (FSL) tools (version 4.1.9) (www.fmrib.ox.ac.uk/fsl) (Smith et al., 2004). Pre-processing using FMR Expert Analysis Tool (FEAT) (version 5.98) involved head-motion correction (Jenkinson et al., 2002), removal of non-brain tissue (Smith, 2002), spatial smoothing (Gaussian 6-mm full width at half-maximum), high-pass temporal filtering $(120 \mathrm{~s})$, affine-registration to the subjects' anatomical T1-weighted scan and subsequently to the Montreal Neurological Institute (MNI) 152 standard space template (Jenkinson and Smith, 2001).

\subsubsection{Independent component analysis}

First, resting state networks in every study subject were identified using a model-free independent component analysis (ICA) approach, multivariate exploratory linear optimised decomposition into independent components (MELODIC) (Beckmann et al., 2005; Beckmann and Smith, 2004, 2005). Spatiotemporal components for each subject were examined, and components that clearly corresponded to noise (e.g., scanner-related or physiological artefacts) were removed (FSL software tool fslregfilt) based on their spatial patterns and temporal frequency characteristics (Beckmann and Smith, 2005), similar to previous studies (de Bie et al., 2012). The filtered and noise-free data were then used for the group analysis. This involved combining all subjects' $(n=68)$ resting state scans into a single 4D data set, which was then decomposed into spatio-temporal components (multi-session temporal concatenation approach) (Beckmann et al., 2005). These component maps were divided by the standard deviation of the residual noise and thresholded at a posterior probability threshold of $p>0.5$ (i.e., an equal loss is placed on false positives and false negatives) by fitting a Gaussian/gamma mixture model to the histogram of intensity values (Beckmann and Smith, 2004). The number of components was restricted to 25 , which has previously been shown to be the optimal number to split fMRI datasets into a final set of spatially independent components (Damoiseaux et al., 2006, 2012). These independent components were inspected visually, and specific networks were identified for further analysis, following spatial correlation against resting state networks previously reported (Beckmann et al., 2005; Smith and Nichols, 2009), i.e., default mode, salience, executive control, basal ganglia and limbic networks (see Fig. 1), as they were expected to be affected in DLB based on the symptom profile.

\subsubsection{Dual regression approach}

For the networks shown in Fig. 1 (identified from the group ICA analysis), functional connectivity differences among DLB, $\mathrm{AD}$ and control subjects were investigated on a voxel-wise basis using a dual regression approach (Filippini et al., 2009; Veer et al. 2010), carried out separately for each independent component, similar to previous reports (Cole et al., 2010; Filippini et al., 2012). This involved the following:

i. Representations of the networks identified in all subjects were created in every individual subject: First regression to extract individual time series associated with each subject and the component of interest followed by a second regression to obtain subject specific maps that were then transformed into $z$ scores.

ii. Assessment of statistical differences between DLB, AD and controls: FSL Randomise (version 2.1) and threshold-free cluster enhancement (TFCE) (Smith and Nichols, 2009) were used to derive separate null distributions of $t$-values for the contrasts reflecting the between- and within-group effects by performing 5000 random permutations and testing the difference between groups or against zero for each iteration (Nichols and Holmes, 2002). Thus, a three-group comparison was carried out to investigate connectivity differences between DLB, AD and controls for each network and the resulting statistical maps thresholded at $p<0.05$ (only family-wise error [FWE] corrected $p$-values $<0.05$ were accepted and thus the chance of one more false positives occurring over space is no more than $5 \%$ and so a $95 \%$ confidence of no false positives in the image). Group comparisons were masked using the network identified from all study subjects (see Supplementary material Fig. 1) so that only differences within the network of interest were investigated (Veer et al., 2010). Brain 


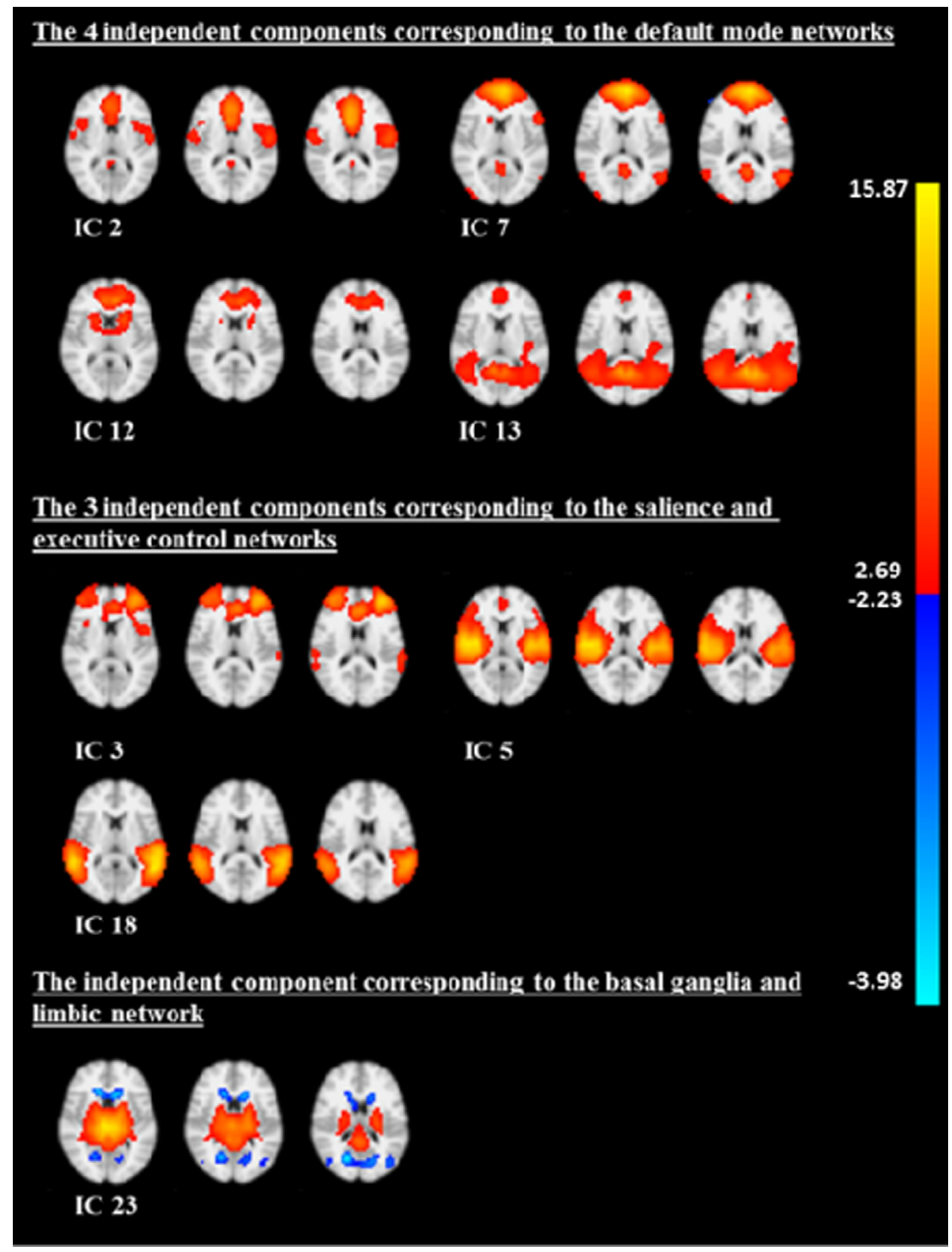

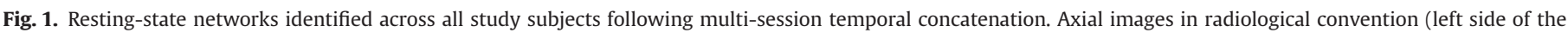
brain $=$ right side of the image as viewed). ICs show $Z$ slices: $21-23, Z$ MNI coordinates: 12,16 , and 20 . Colour bar shows $Z$ statistic scores.

regions showing significant differences between groups were localised using the Talairach atlas in the FSL atlas tools (Talairach and Tournoux, 1988; Lancaster et al., 2000, 2007).

To compare the group demographics, one-way analysis of variance (ANOVA) was used for cross-group comparisons and the independent-samples $t$-test for between-group comparisons $(p<0.05)$ with the Statistical Package for Social Sciences (SPSS) (version 15.0.1) (SPSS for Windows, 2006).

\section{Results}

\subsection{Demographics}

Table 1 shows the clinical characteristics of the study subjects. Groups were similar in age and sex. As would be expected, controls had significantly higher cognitive test scores (MMSE) compared with
DLB and AD subjects. There were no significant differences between DLB and AD subjects for age at onset, duration of dementia, or MMSE scores. In DLB, scores on the UPDRS, NPI, CAFS and GDS were significantly higher compared with $\mathrm{AD}$, indicating greater severity in DLB of the motor features of parkinsonism $(p<0.001)$, neuropsychiatric disturbances $(p<0.001)$, fluctuating cognition $(p=0.027)$ and depressive symptoms $(p=0.003)$, as would be expected based on the known symptom profile of DLB.

At the time of study, 21 subjects were taking acetylcholinesterase inhibitors: $11 \mathrm{AD}$ (donepezil [7 subjects] and galantamine [4 subjects]) and 10 DLB (donepezil [5 subjects], galantamine [4 subjects] and rivastigmine [1 subject]) subjects. No subjects were taking memantine. Seven subjects (6 DLB and 1 AD) were taking antidepressants (citalopram, mirtazapine, trazodone, venlafaxine or paroxetine) and one DLB subject was taking a non-benzodiazepine (zopliclone) as a hypnotic. 
Table 1

Demographic and neuropsychological data of study subjects.

\begin{tabular}{|c|c|c|c|c|c|}
\hline Demographic and neuropsychological data & DLB & $\mathrm{AD}$ & Controls & $p$-value & ANOVA/t-test \\
\hline$N$ & 15 & 13 & 40 & & \\
\hline Age (years) & $80.6 \pm 6.0$ & $75.5 \pm 8.2$ & $77.8 \pm 4.5$ & $0.061^{\mathrm{a}}$ & \\
\hline Sex (M:F) & $9: 6$ & $7: 6$ & $20: 20$ & $0.958^{\mathrm{b}}$ & \\
\hline Age at onset of dementia (years) & $77.2 \pm 6.7$ & $71.9 \pm 8.4$ & & $0.080^{\mathrm{c}}$ & \\
\hline Duration of dementia (months) & $40.2 \pm 20.3$ & $43.0 \pm 22.4$ & & $0.737^{\mathrm{c}}$ & \\
\hline MMSE & $19.5 \pm 4.2$ & $21.5 \pm 3.7$ & $29.1 \pm 1.2$ & $<0.001^{\mathrm{a} *}$ & d.f. $=2, F=88.77$ \\
\hline UPDRS & $22.1 \pm 11.9$ & $6.2 \pm 4.5$ & & $<0.001^{\mathrm{c} *}$ & d.f. $=26, t=4.8$ \\
\hline NPI & $23.1 \pm 11.5$ & $7.0 \pm 6.8$ & & $<0.001^{\mathrm{c} *}$ & d.f. $=21.4, t=4.4$ \\
\hline CAFS & $6.7 \pm 5.3$ & $1.9 \pm 3.8$ & & $0.027^{\mathrm{c} *}$ & d.f. $=22, t=2.4$ \\
\hline GDS & $7.1 \pm 3.3$ & $3.5 \pm 2.4$ & & $0.003^{\mathrm{c} *}$ & d.f. $=26, t=3.3$ \\
\hline
\end{tabular}

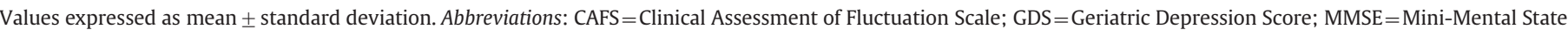
Examination; NPI = Neuropsychiatric Inventory; UPDRS=Unified Parkinson's Disease Rating Scale (subsection III).

- MMSE: Con $>$ AD and DLB $(p<0.001 \text {, d.f. }=2, F=88.77)^{a}$.

- UPDRS: DLB vs. AD $(p<0.001 \text {, d.f. }=26, t=4.8)^{\mathrm{c}}$ (i.e. DLB subjects had greater motor features).

- NPI: DLB vs. $\mathrm{AD}(p<0.001 \text {, d.f. }=21.4, t=4.4)^{\mathrm{c}}$ (i.e. DLB subjects had greater neuropsychiatric symptoms).

- CAFS: DLB vs. $\mathrm{AD}(p=0.027 \text {, d.f. }=22, t=2.4)^{\mathrm{c}}$ (i.e. DLB subjects had greater fluctuations).

- GDS: DLB vs. $\mathrm{AD}(p=0.003 \text {, d.f. }=26, t=3.3)^{\mathrm{c}}$ (i.e. DLB subjects had greater depressive symptoms).

${ }^{\text {a }}$ The $p$-values were calculated using one-way analysis of variance.

${ }^{\mathrm{b}}$ The $p$-value was calculated using the Pearson Chi-Square Test.

${ }^{c}$ The $p$-values were calculated using the independent samples $t$-test.

* The $p$-value is $<0.05$.

\subsection{Functional connectivity results}

\subsubsection{Default mode networks}

Fig. 1 shows the four independent components (ICs) involving brain regions of the default mode network, identified across all study subjects following multi-session temporal concatenation. Peak connectivity clusters were localised to the following areas: cingulate gyrus (IC 2), frontal pole (IC 7), paracingulate gyrus (IC 12) and precuneus cortex (IC 13). ICs 2 and 13 are examined in further detail in Figs. 2 and 3, following dual regression to identify significant differences among DLB, AD and control groups. Functional connectivity was less in DLB compared with AD and controls within a number of brain regions including the precuneus (Fig. 2A and C), inferior parietal (Fig. 2A), middle frontal (Fig. 2C), inferior temporal (Fig. 2A), lingual gyrus (Figs. 2A and 3B) and the posterior lobe of the cerebellum (Fig. $3 \mathrm{~A}$ and $\mathrm{B}$ ). Table 2 summarises the voxels, $p$-values and $t$-statistics for these brain regions. There were no regions of less connectivity in AD subjects compared with control or DLB subjects, or in controls compared with AD subjects. Only one network showed greater connectivity in DLB subjects, and this was compared with controls in superior temporal regions (Fig. 2B). Group comparisons for ICs 7 and 12 are displayed in Supplementary material Figs. 2 and 3 with the brain regions showing significant differences presented in Supplementary Table 1.

\subsubsection{Salience and executive control networks}

Fig. 1 shows the three ICs identified corresponding to brain regions known to be involved in salience and executive control across all study subjects. Peak connectivity clusters were localised to the following areas: frontal pole (IC 3), insular cortex (IC 5) and paracingulate gyrus (IC 18). Figs. 4 and 5 examine frontal (IC 3 ) and insular (IC 5) components in further detail following dual regression to identify significant differences between the DLB, AD and control groups. Functional connectivity was less in the DLB group compared with the $\mathrm{AD}$ and control groups in a number of brain regions including frontal (Figs. $4 \mathrm{~A}$ and $5 \mathrm{~A}$ and $\mathrm{B}$ ), occipital (Fig. 5A), and parietal (Figs. 4A and 5A) cortex. Table 2 summarises the voxels, $p$-values and $t$-statistics for these brain regions. There were no regions of less connectivity in AD subjects compared with control or DLB subjects, or in controls compared with DLB or AD subjects. Group differences for IC 18 are shown in Supplementary material Fig. 4 with the brain regions presented in Supplementary Table 1.

\subsubsection{Basal ganglia and limbic network}

One network was identified across all study subjects corresponding to the basal ganglia and limbic network, with the peak connectivity cluster localised to the thalamus (Fig. 1, IC 23). Fig. 6 shows this network in further detail following dual regression to identify significant differences among the DLB, AD and control groups. Functional connectivity of the basal ganglia and limbic network was affected in DLB subjects only, with functional connectivity greater compared with controls in frontal and limbic regions (Fig. 6A and Table 2). These regions (left superior frontal and left anterior cingulate) were then investigated further by correlating the $z$-statistic score in the peak coordinates (see Table 2 for coordinates) with the UPDRS and fluctuation (CAFS) scores, an analysis that showed that higher UPDRS and CAFS scores (indicating greater severity of symptoms) in DLB significantly correlated with a higher $z$-statistic score (UPDRS: $r=0.625 /$ $p=0.013$, CAFS: $r=0.525 / p=0.045$, see Fig. 7 and Table 3).

Table 4 provides a summary of the above results showing the specific resting state networks investigated in this study and the differences identified among DLB, AD and control subjects.

\section{Discussion}

\subsection{Study findings}

Previous research by our group investigated resting state functional connectivity using a model-based/seed-region approach (Kenny et al., 2011, 2013). This approach was selected because we had prior hypotheses (formed from previous neuroimaging studies) of the regions we expected to be functioning abnormally/ normally in DLB. The disadvantage of that approach is that it only tests connectivity with a predefined brain region. Thus, in the present study, a model-free/ICA approach was used to measure whole brain functional connectivity, an approach that enables comparison in multiple distributed voxels (Cole et al., 2010). 


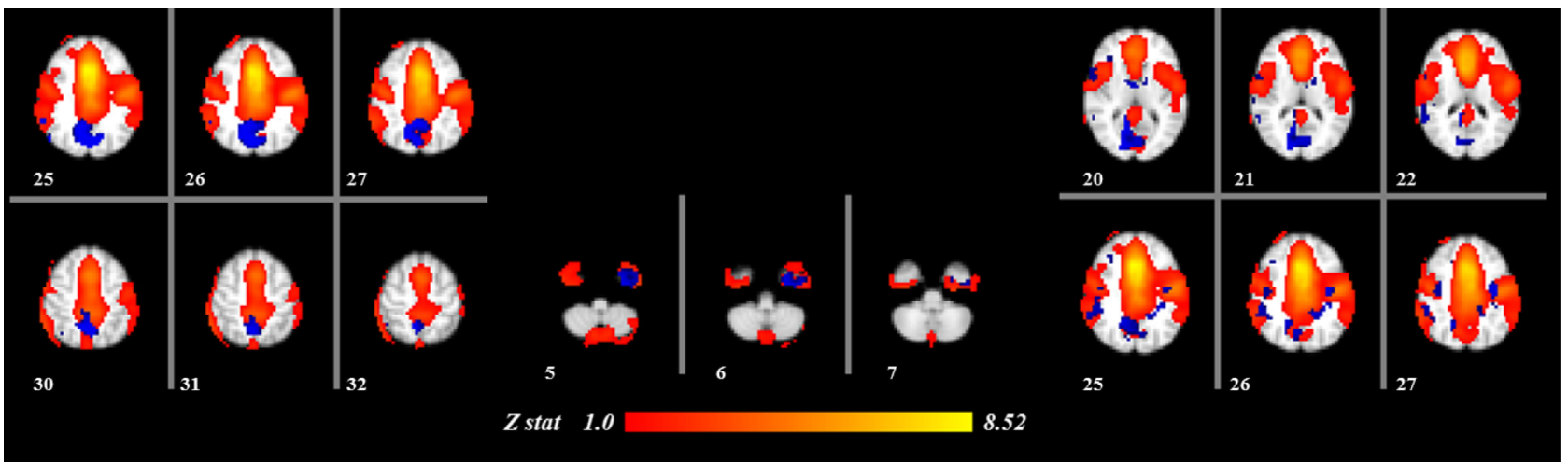

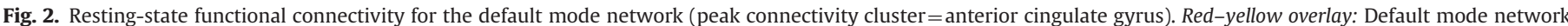

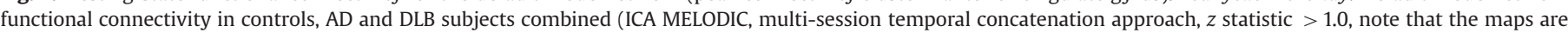

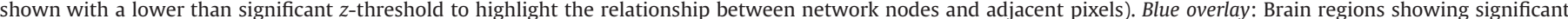

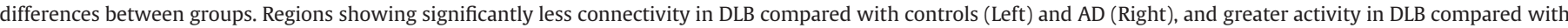

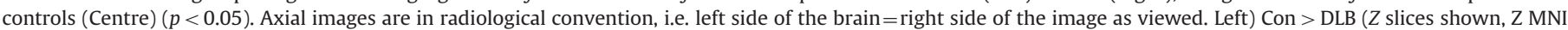

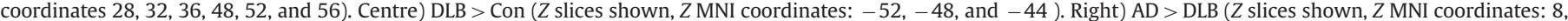
$12,16,28,32$, and 36).

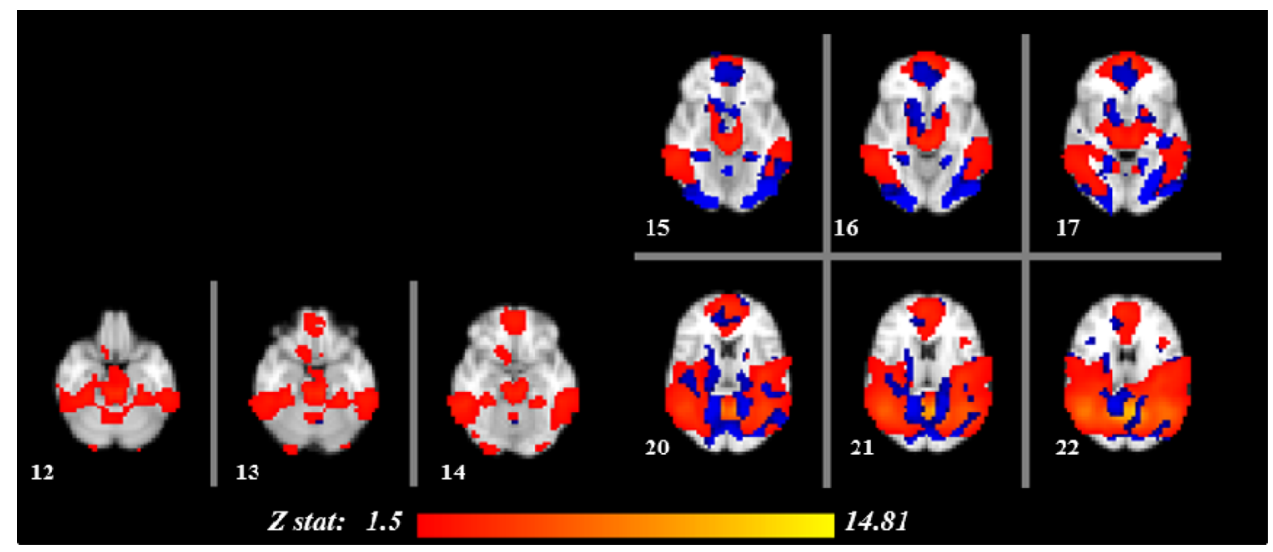

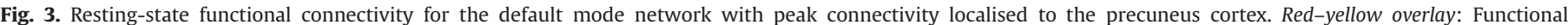

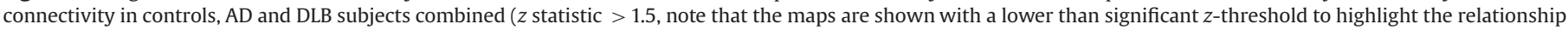

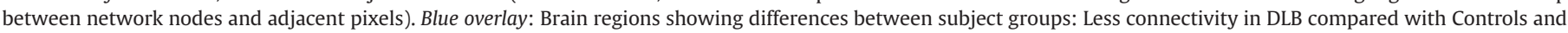

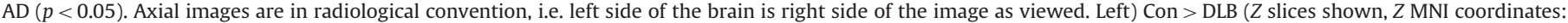
$-24,-20$, and -16$)$. Right) AD $>$ DLB ( $Z$ slices shown, $Z$ MNI coordinates: $-12,-8,-4,8,12$, and 16).

Functional connectivity within specific resting state networks was hypothesised to be more affected in DLB subjects compared with $\mathrm{AD}$ and control subjects based on the symptom profile of DLB.

Default mode, salience and executive control networks were more affected in DLB subjects (functional connectivity generally less compared with control and AD subjects), findings that were as hypothesised based on attention, alertness and executive function deficits in DLB. Similar brain regions showed less connectivity in DLB as follows: precuneus (default mode network), inferior parietal (default mode network), middle frontal (default mode network, salience and executive control), lingual (default mode network, salience and executive control) and postcentral gyri (salience and executive control). In DLB subjects compared with $\mathrm{AD}$ and control subjects, functional connectivity was less in occipital regions (e.g., middle occipital cortex and cuneus) in default mode, salience and executive control networks, which could be related with visual hallucinations, which are a core feature of DLB.

In DLB subjects, the basal ganglia and limbic network showed greater connectivity compared with controls, a difference that could be related to the parkinsonian symptoms and mood disturbances which are common in DLB. Greater connectivity in DLB subjects was only found compared with controls (not AD subjects) and in fewer brain regions-superior temporal (default mode network) and superior frontal and the anterior cingulate cortex (basal ganglia and limbic network)-and generally the cluster size was of a smaller magnitude compared with other networks showing greater connectivity in controls and AD subjects compared with DLB subjects, i.e., 3-8 voxels showing greater connectivity in DLB subjects compared with controls in the basal ganglia network.

In our study the dementia subjects were well matched for age and disease severity; however, we still showed important differences in functional connectivity between DLB and AD subjects for default mode, salience and executive control networks. Functional connectivity was altered in DLB subjects compared with AD and control subjects, whereas in $\mathrm{AD}$ subjects the resting-state networks investigated remained unaffected compared with controls.

\subsection{Previous studies}

We previously showed that basal ganglia regions were more affected in DLB using a different analytic approach (seed region) to investigate connectivity between the caudate and thalamus and all other brain regions (Kenny et al., 2013). Our findings here are entirely consistent with that study in that we also showed greater 
Table 2

Brain regions showing significant differences between groups, corresponding to Figs. 2-6 of the restingstate networks.

\begin{tabular}{|c|c|c|c|c|c|c|}
\hline Corresponding figure and resting state network & Group differences $p<0.05$ & Brain region & Coordinates & Voxels & $p$-value & $\begin{array}{l}t \text {-statistic } \\
\text { (uncorrected) }\end{array}$ \\
\hline \multirow[t]{13}{*}{ Fig. 2: Default mode, cingulate gyrus (anterior) } & \multirow[t]{5}{*}{ A) Con $>$ DLB } & R cuneus/occipital (BA 18) & $6,-78,8$ & 543 & 0.001 & 2.65 \\
\hline & & $\mathrm{R}$ inferior parietal (BA 40) & $62,-42,24$ & 153 & 0.001 & 3.12 \\
\hline & & L caudate head & $-10,10,-4$ & 12 & 0.029 & 3.95 \\
\hline & & R middle occipital (BA 18) & $34,-94,4$ & 9 & 0.004 & 2.84 \\
\hline & & L lingual gyrus (BA 18) & $-14,-54,4$ & 4 & 0.037 & 3.15 \\
\hline & B) $\mathrm{DLB}>$ Con & L superior temporal (BA 38) & $-30,6,-48$ & 81 & 0.012 & 4.25 \\
\hline & \multirow[t]{7}{*}{ C) $\mathrm{AD}>\mathrm{DLB}$} & R precuneus/parietal (BA 7) & $6,-54,40$ & 1067 & 0.004 & 2.67 \\
\hline & & $\mathrm{R}$ thalamus & $6,-6,8$ & 351 & 0.018 & 4.35 \\
\hline & & R precentral gyrus (BA 6) & $42,-6,48$ & 196 & 0.016 & 3.01 \\
\hline & & R subgyral/frontal (BA 8) & $22,30,40$ & 6 & 0.033 & 2.89 \\
\hline & & R middle frontal (BA 9) & $42,34,28$ & 6 & 0.036 & 2.77 \\
\hline & & L middle occipital (BA 18) & $-26,-86,-8$ & 4 & 0.042 & 4.86 \\
\hline & & L middle frontal (BA 6) & $-38,2,52$ & 3 & 0.019 & 2.87 \\
\hline \multirow[t]{5}{*}{ Fig. 3: Default mode, precuneus cortex } & A) Con $>$ DLB & L declive/posterior lobe & $-2,-62,-16$ & 4 & 0.039 & 4.38 \\
\hline & \multirow[t]{4}{*}{ B) $\mathrm{AD}>\mathrm{DLB}$} & L lingual gyrus (BA 18) & $-22,-78,-4$ & 148 & 0.018 & 4.43 \\
\hline & & $\mathrm{R}$ lingual gyrus (BA 18) & $18,-86,-8$ & 42 & 0.025 & 3.31 \\
\hline & & R parahippocampal gyrus (BA 30) & $18,-50,4$ & 29 & 0.043 & 3.46 \\
\hline & & L declive/posterior lobe & $-2,-62,-16$ & 2 & 0.048 & 3.63 \\
\hline \multirow[t]{2}{*}{ Fig. 4: Salience and executive control, frontal pole } & \multirow[t]{2}{*}{ A) $\mathrm{AD}>\mathrm{DLB}$} & R middle frontal (BA 6) & $42,2,48$ & 154 & 0.031 & 3.33 \\
\hline & & R postcentral/parietal (BA 1) & $62,-22,40$ & 18 & 0.031 & 4.41 \\
\hline \multirow[t]{12}{*}{ Fig. 5: Salience and executive control, insular cortex } & \multirow[t]{7}{*}{ A) Con > DLB } & R fusiform gyrus/occipital (BA 19) & $46,-78,-12$ & 57 & 0.014 & 3.60 \\
\hline & & R lingual gyrus/occipital (BA 17) & $22,-102,-8$ & 23 & 0.021 & 3.99 \\
\hline & & L postcentral gyrus/parietal (BA 2) & $-66,-22,36$ & 17 & 0.035 & 3.56 \\
\hline & & R lingual gyrus/occipital (BA 18) & $26,-58,4$ & 7 & 0.029 & 3.19 \\
\hline & & L precentral gyrus/frontal (BA 6) & $-58,-2,48$ & 5 & 0.031 & 3.38 \\
\hline & & L inferior frontal (BA 9) & $-62,22,24$ & 5 & 0.022 & 3.78 \\
\hline & & R middle occipital (BA 19) & $50,-82,8$ & 3 & 0.022 & 3.78 \\
\hline & \multirow{5}{*}{ B) $\mathrm{AD}>\mathrm{DLB}$} & L cingulate (BA 24) & $-2,-2,44$ & 168 & 0.021 & 3.52 \\
\hline & & L precentral gyrus (BA 4) & $-10,-26,68$ & 74 & 0.030 & 3.85 \\
\hline & & $\mathrm{R}$ middle frontal (BA 6) & $38,10,52$ & 22 & 0.036 & 3.53 \\
\hline & & R middle frontal (BA 6) & $22,2,64$ & 7 & 0.041 & 3.35 \\
\hline & & R medial frontal (BA 6) & $6,6,60$ & 7 & 0.037 & 3.04 \\
\hline \multirow[t]{6}{*}{ Fig. 6: Basal ganglia and limbic, thalamus } & \multirow[t]{3}{*}{ A) DLB $>$ Con } & L superior frontal (BA 10) & $-26,54,-8$ & 8 & 0.043 & 4.02 \\
\hline & & $\mathrm{L}$ anterior cingulate/limbic (BA 25) & $-6,18,-16$ & 3 & 0.048 & 2.41 \\
\hline & & L superior frontal (BA 11) & $-22,42,-24$ & 3 & 0.036 & 4.21 \\
\hline & \multirow[t]{3}{*}{ A) $\mathrm{DLB}>$ Con } & L superior frontal (BA 10) & $-26,54,-8$ & 8 & 0.043 & 4.02 \\
\hline & & $\mathrm{L}$ anterior cingulate/limbic (BA 25) & $-6,18,-16$ & 3 & 0.048 & 2.41 \\
\hline & & L superior frontal (BA 11) & $-22,42,-24$ & 3 & 0.036 & 4.21 \\
\hline
\end{tabular}

BA, Brodmann's area.

connectivity in DLB subjects compared with controls in the basal ganglia and limbic network. Greater connectivity of the basal ganglia and limbic network in DLB could explain some of the characteristic symptoms and heightened responses in DLB, which have been linked with regions that form part of this network. For example, greater connectivity of the thalamus could be linked with fluctuations in cognition in DLB as this region is involved in maintenance of consciousness, and it has been shown that DLB subjects with disturbances of consciousness have increased nicotinic receptor binding in the thalamus (Pimlott et al., 2006). Greater connectivity of the caudate in DLB could be linked with depressive symptoms in DLB as this structure is involved with emotional regulation, and in a previous study we showed greater functional connectivity with the caudate in late-life depression compared with controls (Kenny et al., 2010). The putamen is involved in the control of motor functions, and therefore abnormal functioning here and with the networks it forms could be linked with the motor features of parkinsonism which are common in DLB.

In this study we investigated this further by correlating the $z$ statistic of the basal ganglia network regions showing greater connectivity in DLB compared with controls with UPDRS and fluctuation (CAFS) scores in DLB subjects. We showed that higher UPDRS and CAFS scores (indicating greater severity of symptoms) in DLB significantly correlated with a higher $z$-statistic score. Thus, in the DLB subjects with greater symptom severity, the basal ganglia and limbic network were even more affected than in the DLB subjects with less severe symptoms.

In contrast to our findings in this study, we previously showed greater posterior cingulate connectivity in DLB subjects compared with controls (Kenny et al., 2011). This may be explained by the fact that the DLB subjects in our previous study showed connectivity between the posterior cingulate and brain regions (e.g., culmen and cerebellar tonsil) that are not necessarily part of the normal posterior cingulate resting state network; thus, when we look within this network only, these differences are not evident. Although in this study we showed less functional connectivity in DLB subjects compared with $\mathrm{AD}$ and control subjects in the posterior default mode network, this network showed less overall connectivity with different regions, i.e., not the regions where we had found greater connectivity in our previous study, e.g., the declive and lingual gyrus, indicating that the two different analytic approaches were identifying different resting state networks.

To date, there has only been one other resting state study using an ICA approach in DLB, and this only investigated the default mode network (Franciotti et al., 2012). Similar to our findings, Franciotti et al. (2012) also showed reduced functional connectivity in DLB compared with controls. They reported similar affected regions of reduced inferior parietal connectivity in DLB compared with controls. In contrast to our study, they showed less connectivity in $\mathrm{AD}$ compared with controls and no differences in functional connectivity in a direct comparison between AD and 
DLB subjects. However, Franciotti et al. (2012) used a different approach; ICA was used to identify the default mode network, from which key seed regions were identified, the mean BOLD signal time course extracted, and the $z$-score measured to investigate group differences (Franciotti et al., 2012), instead of the dual regression approach used in the present study.

Similar to our findings of less connectivity in DLB, a previous study that investigated resting state functional connectivity in DLB with a model-based/seed-region approach also showed less connectivity in the same regions, specifically the precuneus and occipital regions in DLB compared with control subjects (Galvin et al., 2011). Additionally, Galvin et al. (2011) also showed less

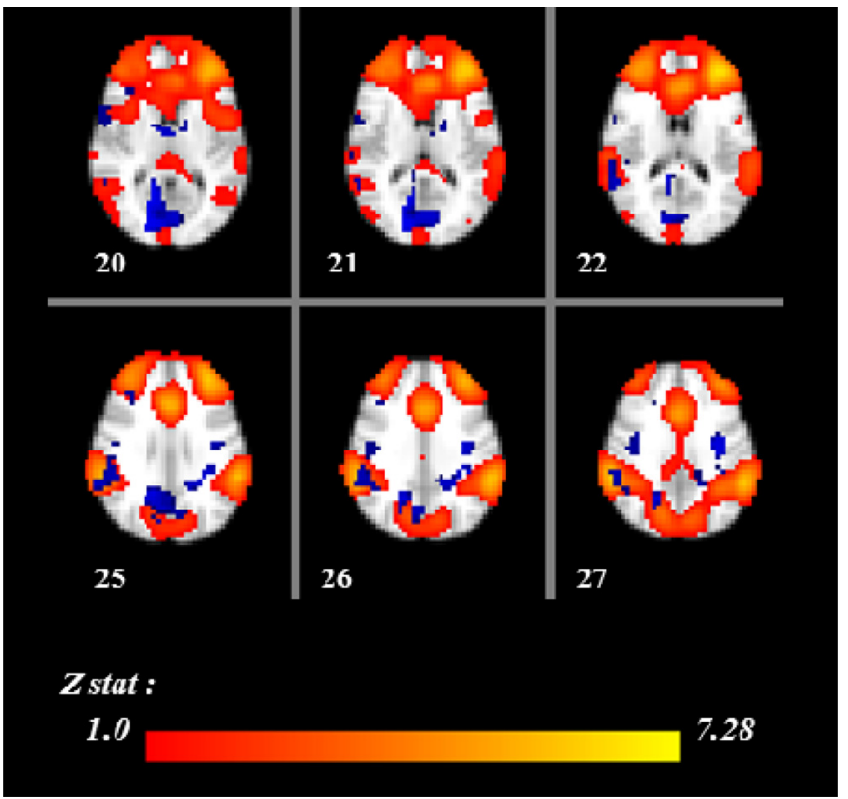

Fig. 4. Resting-state functional connectivity for salience and executive control networks with peak connectivity localised to the frontal pole. Red-yellow overlay: Functional connectivity in all study subjects (controls, AD and DLB subjects combined, $z$ statistic $>1.0$, note that the maps are shown with a lower than significant $z$-threshold to highlight the relationship between network nodes and adjacent pixels). Blue: Regions showing differences between groups, less connectivity in DLB compared with $\mathrm{AD}(p<0.05)$. Axial images in radiological convention left side of brain= right side of image. AD $>$ DLB ( $Z$ slices shown, $Z$ MNI coordinates: $8,12,16,28,32$, and 36 ) connectivity in DLB compared with AD subjects, a finding confirmed in the present study.

Consistent with our findings, previous studies have also shown enhanced connectivity of salience networks in AD (Zhou et al., 2010). This network is involved in social-emotional and visceral autonomic processing (Seeley et al., 2007), and it has been shown to anti-correlate with the default mode network (Fox et al., 2005). Less connectivity in this network in DLB subjects compared with control and $\mathrm{AD}$ subjects could be related to mood disturbances which are common in DLB. We might have expected to see less functional connectivity in $\mathrm{AD}$ subjects compared with controls

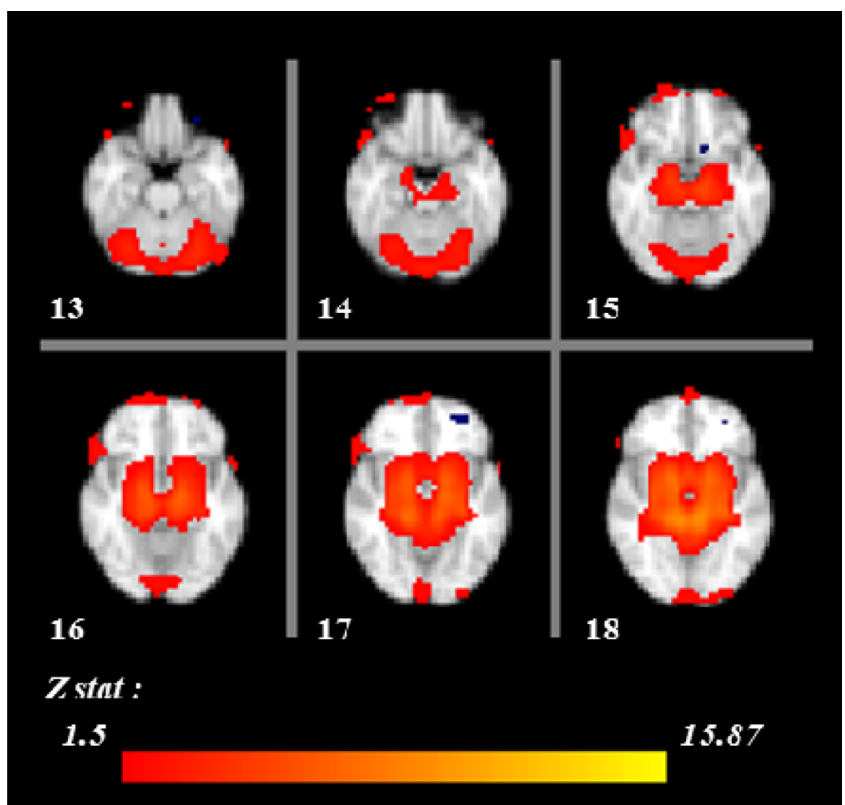

Fig. 6. Resting-state functional connectivity for basal ganglia and limbic network with peak connectivity localised to the thalamus. Red-yellow overlay: Functional connectivity in all study subjects (controls, AD and DLB subjects combined, $z$ statistic $>1.5$, note that the maps are shown with a lower than significant $z$-threshold to highlight the relationship between network nodes and adjacent pixels). Blue overlay: Brain regions showing differences between subject groups, less connectivity in controls compared with DLB $(p<0.05)$. Axial images are in radiological convention, i.e. left side of the brain is right side of the image as viewed. DLB $>$ Con $(Z$ slices shown, $Z$ MNI coordinates: $-20,-16,-12,-8,-4$, and 0 ).

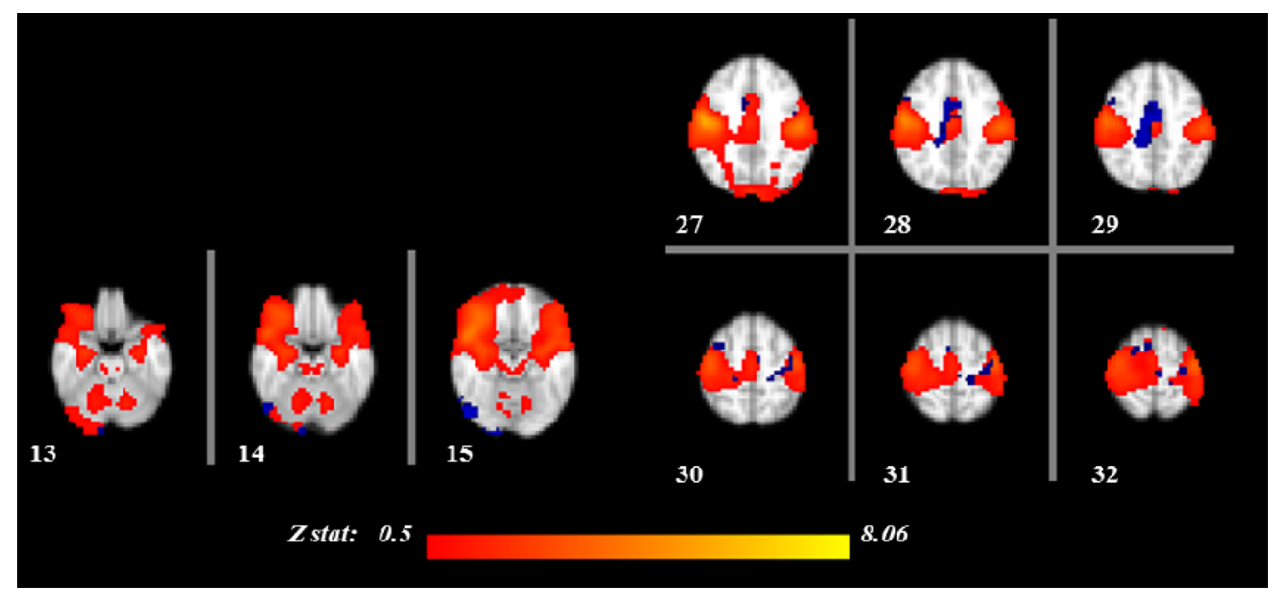

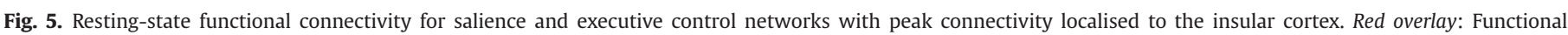

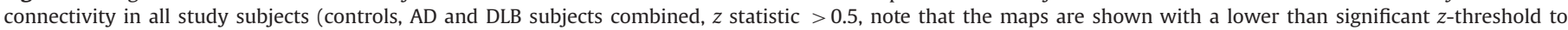

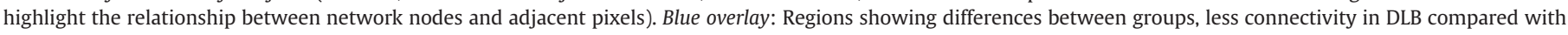

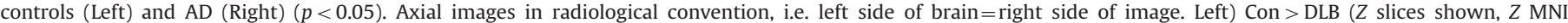
coordinates: $-20,-16$, and -12 ). Right) AD > DLB ( $Z$ slices shown, $Z$ MNI coordinates: $36,40,44,48,52$, and 56 ). 

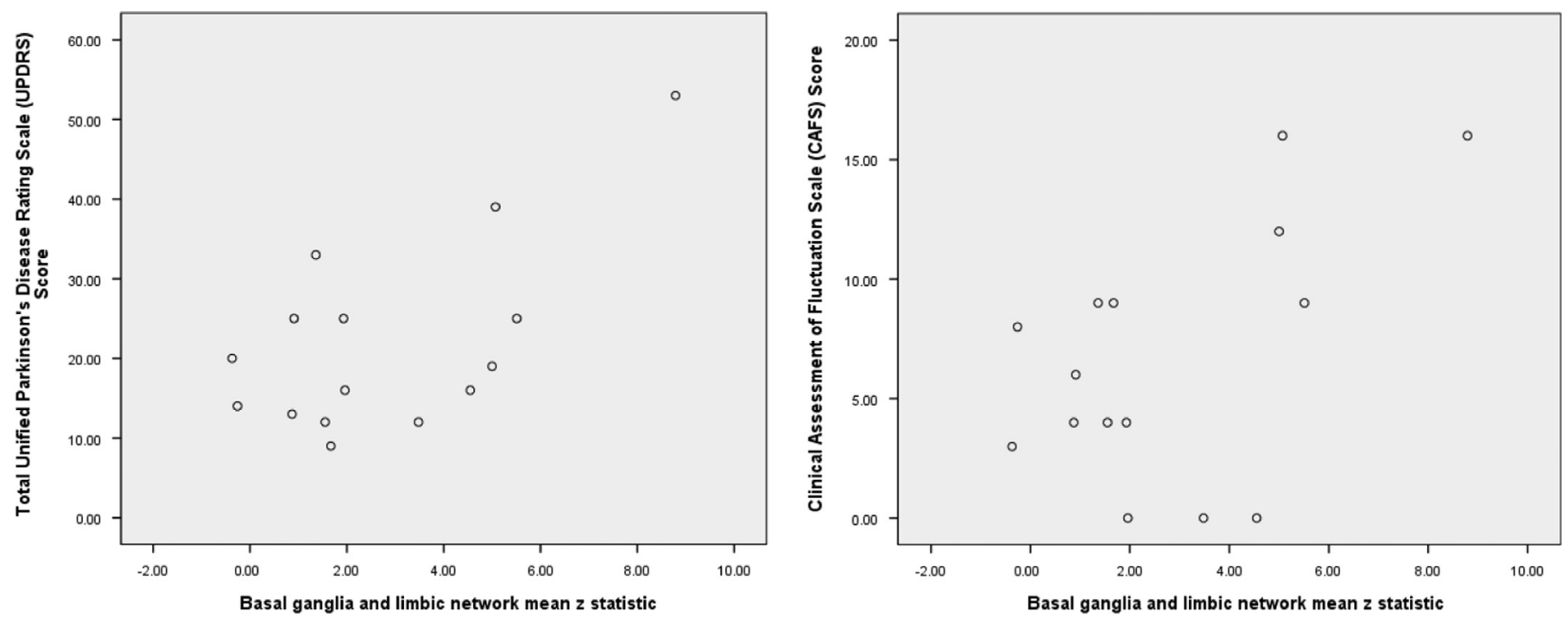

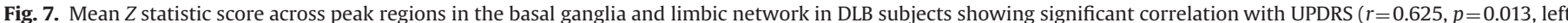
figure) and CAFS ( $r=0.525, p=0.045$, right figure) scores.

Table 3

Mean $z$-statistic score across peak regions in the basal ganglia and limbic network in DLB subjects showing significant correlation with UPDRS and CAFS scores.

\begin{tabular}{|c|c|c|c|c|c|c|c|}
\hline DLB subjects & UPDRS score & CAFS score & $\begin{array}{l}\text { Basal ganglia network } \\
\text { (mean } z \text {-statistic) }\end{array}$ & DLB subjects & UPDRS score & CAFS score & $\begin{array}{l}\text { Basal ganglia network } \\
\text { (mean } z \text {-statistic) }\end{array}$ \\
\hline 1 & 12 & 0 & 3.48 & 10 & 12 & 4 & 1.55 \\
\hline 2 & 53 & 16 & 8.79 & 11 & 25 & 4 & 1.93 \\
\hline 3 & 19 & 12 & 5.00 & 12 & 9 & 9 & 1.67 \\
\hline 4 & 13 & 4 & 0.87 & 13 & 16 & 0 & 1.96 \\
\hline 5 & 33 & 9 & 1.36 & 14 & 25 & 9 & 5.51 \\
\hline 6 & 39 & 16 & 5.07 & 15 & 16 & 0 & 4.55 \\
\hline 7 & 25 & 6 & 0.91 & & & & \\
\hline 8 & 14 & 8 & -0.26 & & & & \\
\hline 9 & 20 & 3 & -0.37 & & & & \\
\hline
\end{tabular}

Table 4

Summary of resting state networks investigated and any significant differences identified between groups.

\begin{tabular}{ll}
\hline Resting state networks & Group differences \\
\hline Default mode networks & \\
Fig. 2: Cingulate gyrus (anterior division) & Con $>$ DLB; DLB $>$ Con; AD $>$ DLB \\
Fig. 3: Precuneus cortex & Con $>$ DLB; AD $>$ DLB \\
Salience and executive control networks & \\
Fig. 4: Frontal pole & AD $>$ DLB \\
Fig. 5: Insular cortex & Con $>$ DLB; AD $>$ DLB \\
Basal ganglia and limbic networks & DLB $>$ Con \\
Fig. 6: Thalamus &
\end{tabular}

within the posterior default mode network as shown in previous studies (Damoiseaux et al., 2012), but no such finding emerged in the present study. Differences between our study and others could be caused by a number of factors, e.g., differences in dementia duration, severity of the disease and age of subjects, thus making it difficult to directly compare studies. Our findings in AD could be a compensatory effect; for example, Damoiseaux et al. (2012) in the same study also showed increased connectivity in other default mode networks, but at follow-up with disease progression there was a loss in connectivity in all networks. Therefore, we may be seeing a compensatory effect in $\mathrm{AD}$ (greater connectivity in some networks/regions to compensate for poor functioning of other networks/regions) and future work looking at disease progression may highlight different changes. Similar to Damoiseaux et al.
(2012), we also showed greater connectivity of the anterior default mode network in $\mathrm{AD}$.

\subsection{Strengths and limitations}

Strengths of this study were that the DLB and AD groups were subject to a thorough diagnosis by consensus between two experienced clinicians. The dementia groups were well matched for onset age, duration of illness, MMSE score. This study used a model-free analytic approach which is advantageous as it allows whole brain functional connectivity to be investigated, whereas when using model-based/seed-region approaches, connectivity can be missed if a brain region does not show direct connectivity with the seed. Model-free methods do not require predefined seeds or a temporal model, and their lack of specificity can make results difficult to interpret. To correct for any potential lack of specificity in this study, we investigated connectivity only within specific resting state networks that we had hypothesised may be more affected in DLB based on the symptoms of disturbances in attention and alertness, executive dysfunction, spontaneous motor features of parkinsonism and depression in DLB. Resting state studies are advantageous as no task has to be practised or performed, which is particularly beneficial for studies involving cognitively impaired subjects for whom it may be more difficult to adhere to a task. Also, in the resting state approach, time in the scanner for the subject is kept to a minimum.

Fluctuations of non-neuronal origin (e.g. cardiac (Chang and Glover, 2009) and respiratory (Wise et al., 2004; Birn et al., 2006) related) can affect resting state data by also correlating in grey matter brain regions and, as they are non-neuronal, they should be 
removed from the data. The analytic approach we used in this study produces noise components separate from the components of interest, and therefore connectivity patterns are not influenced. The resting state is difficult to control, as it can vary greatly between subjects depending on how active a subject's brain is at rest and what the subject is thinking at the time of scanning, but by the same token how well a subject adheres to a task can be variable.

Other resting state networks might be affected in DLB and AD which we did not investigate here, and in future work it will be important to investigate these networks also in relation to specific symptoms in these disorders. Additionally, future work could investigate whether functional connectivity changes are associated with loss in structural volumes in AD and DLB or whether functional connectivity measures relate to processes beyond structural degeneration.

There is currently no definitive clinical diagnosis for DLB or AD, so the validity of diagnostic groups cannot be confirmed until autopsy. However, the clinical diagnostic criteria used in this study have been well validated against autopsy diagnosis (McKeith et al., 2000). More recently, criteria have been developed which show that the combined use of cerebrospinal fluid and imaging (structural/functional/ metabolic) biomarkers can improve the accuracy of the antemortem diagnosis of AD (Jack et al., 2011; McKhann et al., 2011).

The dementia subjects in this study were not medication-free and potential effects on connectivity cannot be ruled out. Previous studies have shown that $\mathrm{AD}$ subjects taking memantine exhibit increased connectivity compared with $\mathrm{AD}$ subjects not taking memantine (Lorenzi et al., 2011); in this study, however, no dementia subjects were taking memantine. Additionally, we identified different patterns of connectivity and significant differences between the dementia groups (and both groups were taking medication), which provides support for the findings not being medication-related. However, potential medication effects cannot be ruled out and, to investigate this further, a longitudinal study of subjects before and after medication would be required.

In conclusion, the main findings of this study were lower functional connectivity in default mode, salience and executive control networks in DLB subjects compared with AD and control subjects, and greater connectivity in the anterior default mode and basal ganglia networks in DLB subjects compared with controls. These findings may aid in greater understanding of what factors underlie the symptom profile of DLB.

\section{Acknowledgements}

The authors thank the study participants for their invaluable contribution and the North East Dementia and Neurodegenerative Diseases Research Network (DeNDRoN) for help with subject recruitment. This work was supported by the National Institute for Health Research (NIHR) Newcastle Biomedical Research Unit and the NIHR Newcastle Biomedical Research Centre for Ageing and Age-related Disease based at Newcastle Hospitals Foundation Trust and Newcastle University, the Biomedical Research Centre and Unit in Dementia award to Cambridge University Hospitals NHS Foundation Trust and the University of Cambridge, the Medical Research Council and Alzheimer's Research UK.

\section{Appendix A. Supplementary material}

Supplementary data associated with this article can be found in the online version at http://dx.doi.org/10.1016/j.pscychresns.2014.06.004

\section{References}

Ballard, C., O'Brien, J., Gray, A., Cormack, F., Ayre, G., Rowan, E., Thompson, P., Bucks, R., McKeith, I., Walker, M., Tovee, M., 2001. Attention and fluctuating attention in patients with dementia with Lewy bodies and Alzheimer disease. Archives of Neurology 58, 977-982.

Beckmann, C.F., DeLuca, M., Devlin, J.T., Smith, S.M., 2005. Investigations into resting-state connectivity using independent component analysis. Philosophical Transactions of the Royal Society of London 360, 1001-1013.

Beckmann, C.F., Smith, S.M., 2004. Probabilistic independent component analysis for functional magnetic resonance imaging. IEEE Transactions in Medical Imaging 23, 137-152.

Beckmann, C.F., Smith, S.M., 2005. Tensorial extensions of independent component analysis for multisubject FMRI analysis. NeuroImage 25, 294-311.

Birn, R.M., Diamond, J.B., Smith, M.A., Bandettini, P.A., 2006. Separating respiratoryvariation-related fluctuations from neuronal-activity-related fluctuations in fMRI. NeuroImage 31, 1536-1548.

Biswal, B., Yetkin, F.Z., Haughton, V.M., Hyde, J.S., 1995. Functional connectivity in the motor cortex of resting human brain using echo-planar MRI. Magnetic Resonance in Medicine 34, 537-541.

Brier, M.R., Thomas, J.B., Snyder, A.Z., Benzinger, T.L., Zhang, D., Raichle, M.E., Holtzman, D.M., Morris, J.C., Ances, B.M., 2012. Loss of intranetwork and internetwork resting state functional connections with Alzheimer's disease progression. Journal of Neuroscience 32, 8890-8899.

Chang, C., Glover, G.H., 2009. Effects of model-based physiological noise correction on default mode network anti-correlations and correlations. NeuroImage 47. 1448-1459.

Cole, D.M., Smith, S.M., Beckmann, C.F., 2010. Advances and pitfalls in the analysis and interpretation of resting-state fMRI data. Frontiers in Systems Neuroscience 4,8 .

Cummings, J.L., Mega, M., Gray, K., Rosenberg-Thompson, S., Carusi, D.A., Gornbein, J., 1994. The Neuropsychiatric Inventory: comprehensive assessment of psychopathology in dementia. Neurology 44, 2308-2314.

Damoiseaux, J.S., Prater, K.E., Miller, B.L., Greicius, M.D., 2012. Functional connectivity tracks clinical deterioration in Alzheimer's disease. Neurobiology of Aging 33 (4), 828.e19-30.

Damoiseaux, J.S., Rombouts, S.A., Barkhof, F., Scheltens, P., Stam, C.J., Smith, S.M., Beckmann, C.F., 2006. Consistent resting-state networks across healthy subjects. Proceedings of the National Academy of Sciences of the United States of America 103, 13848-13853.

de Bie, H.M.A., Boersma, M., Adriaanse, S., Veltman, D.J., Wink, A.M., Roosendaal, S.D., Barkhof, F., Stam, C.J., Oostrom, K.J., Delemarre-van de Waal, H.A., Sanz-Arigita, E.J., 2012. Resting-state networks in awake five- to eight-year old children. Human Brain Mapping 33, 1189-1201.

Fahn, S., Elton, R., 1987. Members of the UPDRS Development Committee: Unified Parkinson's Disease Rating Scale. In: Fahn, S., Marsden, C.D., Calne, D.B., Goldstein, D.R. (Eds.), Recent Developments in Parkinson's Disease. MacMillan Heathcare Information, Florham Park, NJ.

Filippini, N., MacIntosh, B.J., Hough, M.G., Goodwin, G.M., Frisoni, G.B., Smith, S.M., Matthews, P.M., Beckmann, C.F., Mackay, C.E., 2009. Distinct patterns of brain activity in young carriers of the APOE-epsilon4 allele. Proceedings of the National Academy of Sciences of the United States of America 106, 7209-7214.

Filippini, N., Nickerson, L.D., Beckmann, C.F., Ebmeier, K.P., Frisoni, G.B., Matthews, P.M. Smith, S.M., Mackay, C.E., 2012. Age-related adaptations of brain function during a memory task are also present at rest. NeuroImage 59, 3821-3828.

Folstein, M.F., Folstein, S.E., McHugh, P.R., 1975. "Mini-mental state". A practical method for grading the cognitive state of patients for the clinician. Journal of Psychiatric Research 12, 189-198.

Fox, M.D., Raichle, M.E., 2007. Spontaneous fluctuations in brain activity observed with functional magnetic resonance imaging. Nature Reviews 8, 700-711.

Fox, M.D., Snyder, A.Z., Vincent, J.L., Corbetta, M., Van Essen, D.C., Raichle, M.E., 2005. The human brain is intrinsically organized into dynamic, anticorrelated functional networks. Proceedings of the National Academy of Sciences of the United States of America 102, 9673-9678.

Franciotti, R., Falasca, N.W., Bonanni, L., Anzellotti, F., Maruotti, V., Comani, S., Thomas, A., Tartaro, A., Taylor, J.P., Onofrj, M., 2012. Default network is not hypoactive in dementia with fluctuating cognition: an Alzheimer disease/ dementia with Lewy bodies comparison. Neurobiology of Aging 34, 1148-1158.

Galvin, J.E., Price, J.L., Yan, Z., Morris, J.C., Sheline, Y.I., 2011. Resting bold fMRI differentiates dementia with Lewy bodies vs Alzheimer disease. Neurology 76, 1797-1803.

Geser, F., Wenning, G.K., Poewe, W., McKeith, I., 2005. How to diagnose dementia with Lewy bodies: state of the art. Movement Disorders 20 (Suppl 12), S11-S20.

Jack Jr., C.R., Albert, M.S., Knopman, D.S., McKhann, G.M., Sperling, R.A., Carrillo, M.C. Thies, B., Phelps, C.H., 2011. Introduction to the recommendations from the National Institute on Aging-Alzheimer's Association workgroups on diagnostic guidelines for Alzheimer's disease. Alzheimers \& Dementia 7, 257-262.

Jenkinson, M., Bannister, P., Brady, M., Smith, S., 2002. Improved optimization for the robust and accurate linear registration and motion correction of brain images. NeuroImage 17, 825-841.

Jenkinson, M., Smith, S., 2001. A global optimisation method for robust affine registration of brain images. Medical Image Analysis 5, 143-156.

Kenny, E.R., Blamire, A.M., Firbank, M.J., O’Brien, J.T., 2011. Functional connectivity in cortical regions in dementia with Lewy bodies and Alzheimer's disease. Brain $135,569-581$. 
Kenny, E.R., O’Brien, J.T., Cousins, D.A., Richardson, J., Thomas, A.J., Firbank, M.J., Blamire, A.M., 2010. Functional connectivity in late-life depression using resting-state functional magnetic resonance imaging. American Journal of Geriatric Psychiatry 18, 643-651.

Kenny, E.R., O’Brien, J.T., Firbank, M.J., Blamire, A.M., 2013. Subcortical connectivity in dementia with Lewy bodies and Alzheimer's disease. British Journal of Psychiatry 203 (3), 209-214.

Lancaster, J.L., Tordesillas-Gutierrez, D., Martinez, M., Salinas, F., Evans, A., Zilles, K., Mazziotta, J.C., Fox, P.T., 2007. Bias between MNI and Talairach coordinates analyzed using the ICBM-152 brain template. Human Brain Mapping 28, 1194-1205.

Lancaster, J.L., Woldorff, M.G., Parsons, L.M., Liotti, M., Freitas, C.S., Rainey, L., Kochunov, P.V., Nickerson, D., Mikiten, S.A., Fox, PT, 2000. Automated Talairach atlas labels for functional brain mapping. Human Brain Mapping 10, 120-131.

Lorenzi, M., Beltramello, A., Mercuri, N., Canu, E., Zoccatelli, G., Pizzini, F., Alessandrini, F., Cotelli, M., Rosini, S., Costardi, D., Caltagirone, C., Frisoni, G.B., 2011. Effect of memantine on resting state default mode network activity in Alzheimer's disease. Drugs \& Aging 28, 205-217.

McKeith, I.G., Ballard, C.G., Perry, R.H., Ince, P.G., O’Brien, J.T., Neill, D., Lowery, K., Jaros, E., Barber, R. Thompson, P., Swann, A., Fairbairn, A.F., Perry, E.K., 2000 Prospective validation of Consensus criteria for the diagnosis of dementia with Lewy bodies. Neurology 54, 1050-1058.

McKeith, I.G., Dickson, D.W., Lowe, J., Emre, M., O’Brien, J.T., Feldman, H., Cummings, J. Duda, J.E., Lippa, C., Perry, E.K., Aarsland, D., Arai, H., Ballard, C.G., Boeve, B., Burn, D.J., Costa, D., Del Ser, T., Dubois, B., Galasko, D., Gauthier, S., Goetz, C.G., Gomez-Tortosa, E., Halliday, G., Hansen, L.A., Hardy, J., Iwatsubo, T., Kalaria, R.N., Kaufer, D., Kenny, R.A., Korczyn, A., Kosaka, K., Lee, V.M., Lees, A., Litvan, I., Londos, E., Lopez, O.L., Minoshima, S., Mizuno, Y., Molina, J.A., Mukaetova-Ladinska, E.B., Pasquier, F., Perry, R.H., Schulz, J.B., Trojanowski, J.Q., Yamada, M., 2005. Diagnosis and management of dementia with Lewy bodies: third report of the DLB Consortium. Neurology 65, 1863-1872.

McKeith, I.G., Galasko, D., Kosaka, K., Perry, E.K., Dickson, D.W., Hansen, L.A., Salmon, D.P., Lowe, J., Mirra, S.S., Byrne, E.J., Lennox, G., Quinn, N.P., Edwardson, J.A., Ince, P.G., Bergeron, C., Burns, A., Miller, B.L., Lovestone, S., Collerton, D., Jansen, E.N., Ballard, C., de Vos, R.A., Wilcock, G.K., Jellinger, K.A., Perry, R.H., 1996. Consensus guidelines for the clinical and pathologic diagnosis of dementia with Lewy bodies (DLB): report of the consortium on DLB international workshop. Neurology 47, 1113-1124.

McKhann, G., Drachman, D., Folstein, M., Katzman, R., Price, D., Stadlan, E.M., 1984. Clinical diagnosis of Alzheimer's disease: report of the NINCDS-ADRDA Work Group under the auspices of Department of Health and Human Services Task Force on Alzheimer's Disease. Neurology 34, 939-944.

McKhann, G.M., Knopman, D.S., Chertkow, H., Hyman, B.T., Jack Jr., C.R., Kawas, C.H., Klunk, W.E., Koroshetz, W.J., Manly, J.J., Mayeux, R., Mohs, R.C., Morris, J.C., Rossor, M.N., Scheltens, P., Carrillo, M.C., Thies, B., Weintraub, S., Phelps, C.H., 2011. The diagnosis of dementia due to Alzheimer's disease: recommendations from the National Institute on Aging-Alzheimer's Association workgroups on diagnostic guidelines for Alzheimer's disease. Alzheimers \& Dementia 7 263-269.

Nichols, T.E., Holmes, A.P., 2002. Nonparametric permutation tests for functional neuroimaging: a primer with examples. Human Brain Mapping 15, 1-25.

O’Brien, J.T., Colloby, S., Fenwick, J., Williams, E.D., Firbank, M., Burn, D., Aarsland, D., McKeith, I.G., 2004. Dopamine transporter loss visualized with FP-CIT SPECT in the differential diagnosis of dementia with Lewy bodies. Archives of Neurology 61, 919-925.

Perry, E.K., Perry, R.H., 2004. Neurochemistry of consciousness: cholinergic pathologies in the human brain. Progress in Brain Research 145, 287-299.

Pimlott, S.L., Piggott, M., Ballard, C., McKeith, I., Perry, R., Kometa, S., Owens, J., Wyper, D., Perry, E., 2006. Thalamic nicotinic receptors implicated in disturbed consciousness in dementia with Lewy bodies. Neurobiology of Disease 21, 50-56.

Raichle, M.E., MacLeod, A.M., Snyder, A.Z., Powers, W.J., Gusnard, D.A., Shulman, G.L. 2001. A default mode of brain function. Proceedings of the National Academy of Sciences of the United States of America 98, 676-682.

Seeley, W.W., Menon, V., Schatzberg, A.F., Keller, J., Glover, G.H., Kenna, H., Reiss, A.L. Greicius, M.D., 2007. Dissociable intrinsic connectivity networks for salience processing and executive control. Journal of Neuroscience 27, 2349-2356.

Sheikh, J.I., Yesavage, J.A., 1986. Geriatric Depression Scale (GDS). Recent evidence and development of a shorter version. In: Brink, T.L. (Ed.), Clinical Gerontology: A Guide to Assessment and Intervention. The Haworth Press, Inc., New York, pp. 165-173.

Smith, S.M., 2002. Fast robust automated brain extraction. Human Brain Mapping $17,143-155$.

Smith, S.M., Jenkinson, M., Woolrich, M.W., Beckmann, C.F., Behrens, T.E., JohansenBerg, H., Bannister, P.R., De Luca, M., Drobnjak, I., Flitney, D.E., Niazy, R.K. Saunders, J., Vickers, J., Zhang, Y., De Stefano, N., Brady, J.M., Matthews, P.M. 2004. Advances in functional and structural MR image analysis and implementation as FSL. Neurolmage 23 (Suppl 1), S208-S219.

Smith, S.M., Nichols, T.E., 2009. Threshold-free cluster enhancement: addressing problems of smoothing, threshold dependence and localisation in cluster inference. Neurolmage 44, 83-98.

Talairach, J., Tournoux, P., 1988. Co-planar stereotaxic atlas of the human brain: an approach to medical cerebral imaging. Thieme Medical Publishers, Stuttgart, New York.

Veer, I.M., Beckmann, C.F., van Tol, M.J., Ferrarini, L., Milles, J., Veltman, D.J., Aleman, A., van Buchem, M.A., van der Wee, N.J., Rombouts, S.A., 2010. Whole brain restingstate analysis reveals decreased functional connectivity in major depression Frontiers in Systems Neuroscience 4, 41.

Walker, M.P., Ayre, G.A., Cummings, J.L., Wesnes, K., McKeith, I.G., O’Brien, J.T. Ballard, C.G., 2000. The Clinician Assessment of Fluctuation and the One Day Fluctuation Assessment Scale. Two methods to assess fluctuating confusion in dementia. British Journal of Psychiatry 177, 252-256.

Walker, Z., Costa, D.C., Walker, R.W., Shaw, K., Gacinovic, S., Stevens, T., Livingston, G., Ince, P., McKeith, I.G., Katona, C.L., 2002. Differentiation of dementia with Lewy bodies from Alzheimer's disease using a dopaminergic presynaptic ligand. Journal of Neurology, Neurosurgery and Psychiatry 73, 134-140.

Wise, R.G., Ide, K., Poulin, M.J., Tracey, I., 2004. Resting fluctuations in arteria carbon dioxide induce significant low frequency variations in BOLD signal. NeuroImage 21, 1652-1664.

Zhang, Z., Liu, Y., Jiang, T., Zhou, B., An, N., Dai, H., Wang, P., Niu, Y., Wang, L., Zhang, X. 2011. Altered spontaneous activity in Alzheimer's disease and mild cognitive impairment revealed by regional homogeneity. NeuroImage 59, 1429-1440.

Zhou, J., Greicius, M.D., Gennatas, E.D., Growdon, M.E., Jang, J.Y., Rabinovici, G.D. Kramer, J.H., Weiner, M., Miller, B.L., Seeley, W.W., 2010. Divergent network connectivity changes in behavioural variant frontotemporal dementia and Alzheimer's disease. Brain 133, 1352-1367.

SPSS for Windows, 2006. SPSS. Inc., Chicago, IL. 